\title{
Initial dynamic thermal dissipation modes enhance heat dissipation in gold nanoparticle-polydimethylsiloxane thin films
}

\author{
Tyler V. Howard ${ }^{1} \cdot$ Keith R. Berry Jr. ${ }^{2}$ (D) D. Keith Roper ${ }^{3}$ (I)
}

Received: 18 July 2019 / Accepted: 13 January 2020 / Published online: 7 February 2020

(C) The Author(s) 2020

\begin{abstract}
Plasmonic nanocomposite materials have exhibited value for applications ranging from biological hyperthermia to optical sensing and waveguiding. Energy absorbed from incident irradiation can be re-emitted as light or decay into phonons that propagate through the surrounding material and increase its temperature. Previous works have examined steady-state thermal dissipation resulting from irradiated plasmonic nanocomposites. This work shows heat dissipation in the first few seconds can significantly exceed that during subsequent steady state, depending on film geometry, nanoparticle diameter and concentration, laser irradiation power, and position within and adjacent to the irradiated spot. Films of lower thickness containing $16 \mathrm{~nm}$ gold nanoparticles (AuNPs) irradiated at $13.5 \mathrm{~mW}$ laser power showed highest enhancement and tunability of the dynamic thermal mode within and adjacent to the irradiated spot. Measured initial nanocomposite film temperature in or near the irradiated spot exceeded that resulting from constant bulk film thermal dissipation. These results improve understanding of cooling dynamics of resonantly irradiated nanocomposite materials and guide development of devices with enhanced thermal dissipation dynamics.
\end{abstract}

Keywords Nanocomposite films · Optothermal heating · Thermal dissipation

\section{Introduction}

Plasmonically active materials containing various nanostructures have grown in interest in recent years, due to their ability to redistribute electromagnetic energy into different forms (i.e., re-emission, scattering, phonons, polaritons) [1-3]. Energy from incident radiation couples with the electron cloud of the material, is reradiated and decays into phonons, before relaxing into the surrounding medium

Electronic supplementary material The online version of this article (https://doi.org/10.1007/s10973-020-09331-5) contains supplementary material, which is available to authorized users.

Tyler V. Howard and Keith R. Berry Jr have contributed equally.

D. Keith Roper

Keith.Roper@usu.edu

1 Institute of Optics, University of Rochester, Rochester, NY 14627, USA

2 Department of Electrical and Computer Engineering, Baylor University, Waco, TX 76710, USA

3 Department of Biological Engineering, Utah State University, Logan, UT 84322, USA
$[4,5]$. These optical and thermal characteristics have been used in materials that facilitate increased performance in catalytic reactions [6], thermal ablation [7], sensing [8], and solar cells $[9,10]$. Substrate composition [11], nanoparticle composition $[12,13]$ and morphology of plasmonic metamaterial [14, 15] along with intensity of incident light promote tunability of metamaterials containing plasmonic nanostructures $[16,17]$.

Steady-state heat dissipation due to the photothermal responses of plasmonic nanoparticles (NPs) has been widely characterized in various media like colloidal suspensions [6], polymers [1], and ceramics [18]. NPs dispersed in colloidal suspensions of various fluids (i.e., nanofluids) resulted in an increase in the thermal conductivity of the suspension [19], but the thermal response per NP decreased to near zero [20]. Numerical models for estimating and modeling the heat dissipation have also been used to analyze nanofluids used in microchannels [21], while other models utilize the thermodynamic properties to predict the structure of materials $[22,23]$. Heat exchangers and other thermodynamic components have recently been constructed using NP-containing materials in order to analyze the heat transfer properties of the materials and components [24]. These steady-state heat 
dissipation studies have advanced understanding of thermal responses of plasmonically active nanocomposite materials. This has been useful, for example, to interpret heating in a biological microscope [25]. While a linear, continuum description of steady-state heat dissipation has been largely used to characterize plasmonic heat transfer, understanding of dynamic changes in thermal dissipation that occur in resonantly irradiated nanocomposite matter is still in its infancy.

This work showed that dynamically changing heat dissipation from optoplasmonically-energized gold (Au) NPs randomly dispersed into polydimethylsiloxane (PDMS) thin films enhanced the thermal dissipation in and adjacent to the laser-irradiated spot in the first seconds after laser irradiation ceased. Key variables that influenced the degree of enhancement included film geometry, AuNP diameter and concentration, laser irradiation power, and radial distance of the pixel from the laser spot. In contrast to steady-state heat dissipation, in which the temperature driving force for heat transfer is observed to decay logarithmically with time, enhancement during the initial period of dynamic heat dissipation exhibited superlinear decay in the driving force, eventually reaching logarithmic decay within several seconds. The enhancement in heat transfer was observed to be highest for $16 \mathrm{~nm}$ AuNPs in thinner polymer films at $13.5 \mathrm{~mW}$ laser irradiation for nanocomposite in pixels within and adjacent to the laser-heated spot. Understanding of this early dynamic period of optoplasmonic thermal decay in insulative media is anticipated to improve the accuracy of thermal dynamic simulations and enhance the design and implementation of plasmonically active nanoparticle-containing media in various heat transfer scenarios.

\section{Materials and methods}

\section{Film fabrication and AuNP addition}

Thin PDMS films containing AuNPs were fabricated using a recent method that increased NP content and eliminated observable gas/vapor entrapment and NP aggregation [26]. Briefly, AuNP-PDMS films were fabricated as follows. For $16 \mathrm{~nm}$ AuNP films, dried, $16 \mathrm{~nm}$ poly(vinylpyrrolidone) (PVP)-coated AuNPs (Nanocomposix Inc., San Diego, CA) were dispersed into isopropanol (IPA) to $1 \mathrm{mg} \mathrm{mL}^{-1}$ and then dispersed into PDMS (Sylgard ${ }^{\circledR} 184$ silicone elastomer kit \#4019862, Dow Corning, Midland, MI) at $10^{11}-10^{12}$ $\mathrm{NP} \mathrm{cm}{ }^{-3}$. The process consisted of four steps. (1) PDMS monomer and cross-linker were mixed at a 10:1 ratio and degassed for 2 h. (2) A volume of AuNP-IPA dispersion calculated to achieve the desired AuNP content was added into the uncured PDMS and mixed for $1 \mathrm{~h}$. (3) A $1.5 \mathrm{~g}$ mass of AuNP-IPA-PDMS was poured into $25 \times 25 \times 1 \mathrm{~mm}$ polystyrene sample box. (4) The sample box was wrapped in foil to prevent light-induced aggregation and cured at $60{ }^{\circ} \mathrm{C}$ for $24 \mathrm{~h}$. It was previously observed that lowering light incident on suspended AuNPs reduced their aggregation. Such aggregates exhibit reduced optical absorption and optothermal transduction efficiency [16]. The $16 \mathrm{~nm}$ AuNP-containing films are shown in Fig. 1. Fabrication of $76 \mathrm{~nm}$ AuNP-PDMS films followed this procedure but used $76 \mathrm{~nm}$ poly(vinylpyrrolidone) (PVP)-coated AuNPs (Nanocomposix Inc., San Diego, CA) instead of $16 \mathrm{~nm}$ AuNPs. Images of the resulting $76 \mathrm{~nm}$ AuNP-PDMS films are shown in Figure $\mathrm{S} 1$ in Supporting Information. This curing process could be used for PVP-coated AuNPs of any size dispersed in IPA.

\section{Optical analysis}

Spectra of the AuNP-PDMS films fabricated using the method above were captured using a spectrometer (AvaSpec-2048, Avantes, Broomfield, CO, USA). Optical properties (i.e., scattering, absorption, and extinction) of AuNP were derived via Mie theory (Mie Theory Calculator, nanoComposix, San Diego, CA, USA). Mie theory calculates the scattering, absorption and extinction of an isolated particle.

\section{Thermal characterization}

AuNP-PDMS films fabricated using the above method were irradiated resonantly using a $532 \mathrm{~nm}$ laser (MXL-FN-532, CNI, Changchun, China) at $13.5 \mathrm{~mW}$ and $25 \mathrm{~mW}$, as shown in Scheme 1. Laser power was recorded using a power meter (PM310D, Thorlabs, Newton, NJ). Infrared images of the AuNP-PDMS nanocomposites were captured at five images per second over 6 minutes (min): 0:10 min ambient conditions, 2:50 min laser irradiation, and 3:00 min cooling using an infrared thermal imaging camera (ICI 7320, Infrared Cameras Inc., Beaumont, TX). A laser shutter (SH05, Thorlabs, Newton, NJ) and shutter controller (SC10, Thorlabs, Newton, NJ) were used to open and close laser at each time interval. Each AuNP-PDMS film was mounted with tweezers to position the incident laser spot at the film top center. All power incident on the samples was contained

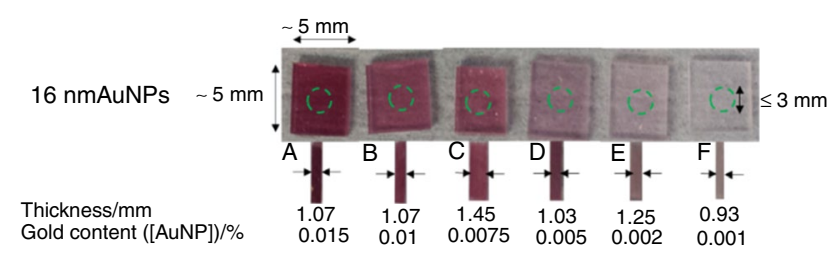

Fig. 1 Images of the PDMS films containing $16 \mathrm{~nm}$ AuNPs showing corresponding concentrations, dimensions, and laser spot size and position 


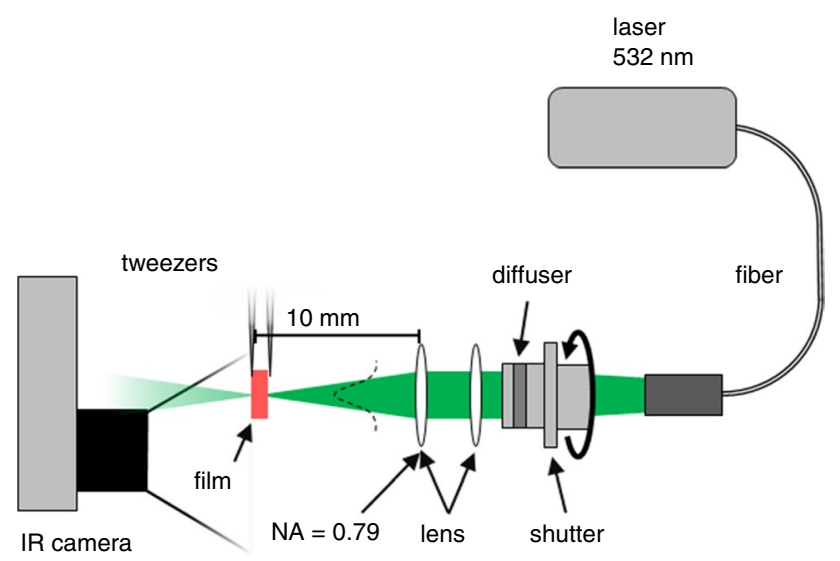

Scheme 1 Optical setup of laser irradiation system with $532 \mathrm{~nm}$ light source. Laser light passed through a manual shutter and $10^{\circ}$ ground glass diffuser to ensure a Gaussian beam profile. New beam profile was then focused to $\leq 3 \mathrm{~mm}$ focal point $10 \mathrm{~mm}$ away from the last lens. Resulting thermal response from AuNP-PDMS films was captured via infrared (IR) camera

within a laser spot with a diameter of $\leq 3 \mathrm{~mm}$. The thermal measurement apparatus was isolated from ambient light and forced convection by enclosure in an opaque chamber. Two or more replicates were measured.

\section{Numerical methods for quantitative and qualitative analysis}

Thermal images captured during optothermal characterization were used to produce thermal micrographs on a pixelby-pixel basis using MATLAB R2018a (MathWorks, Natick, MA). All subsequent analysis and image processing were performed using MATLAB. The thermal micrographs of immediate temperature changes were extracted from the raw thermal images and divided into pixelated, $0.212 \mathrm{~cm} /$ pixel for $16 \mathrm{~nm}$ AuNP at $13.5 \mathrm{~mW}$ and $0.116 \mathrm{~cm} /$ pixel for 16 and $76 \mathrm{~nm}$ AuNP at $25 \mathrm{~mW}$, temperature heat maps. Pixel resolution was optimized using the ICI 7320 camera, as illustrated in Scheme 1, to minimize optical interference and photodamage while preserving pixel uniformity across the sample. Pixel resolution was derived from a calibration standard adjacent to the sample and imaging the sample with the camera set to maximum zoom. Maximum zoom of the infrared camera was selected to minimize effects of diffuse scattered light on camera images. Observations of heat flow from the heat spot were shown as the change in temperature $1 \mathrm{~s}$ before $\left(T\left(1^{-}\right)\right)$and $1 \mathrm{~s}$ after $\left(T\left(1^{+}\right)\right)$laser shutoff (i.e., $\left.\Delta T=T\left(1^{+}\right)-T\left(1^{-}\right)\right)$.

Spatial gradient of change in temperature $(S)$ was constructed to display rate of thermal dissipation across the PDMS films. Gradients from the change in the temperature micrograph were calculated by taking half of the difference from adjacent cells, while cells on the edge were solely the difference with the adjacent cell. Gradients were calculated in the $x$-(column) and $y$-(row) directions for each change in temperature graph and magnitude determined using Eq. (1) shown below:

$S=\frac{\sqrt{\Delta T_{\mathrm{x}}^{2}+\Delta T_{\mathrm{y}}^{2}}}{\max \left(\sqrt{\Delta T_{\mathrm{x}}^{2}+\Delta T_{\mathrm{y}}^{2}}\right)}$

where $\Delta T_{\mathrm{x}}$ is the gradient in the ' $x$ '-direction, $\Delta T_{\mathrm{y}}$ is the gradient in the ' $y$ '-direction, and $\max (\ldots)$ is the absolute maximum magnitude of the gradient in a cell across all films.

Regions of interest consisting of $3 \times 3$ pixels for $13.5 \mathrm{~mW}$ and $9 \times 9$ for $25 \mathrm{~mW}$ were chosen for comparison of the thermodynamic properties across the film. Region of interest for each thermal trial was initially selected to encompass the laser irradiation spot. Calculated thermal behavior for each film was determined via a dimensionless temperature driving force (theta), $\theta=\left(T_{\mathrm{amb}}-T\right) /\left(T_{\mathrm{amb}}-T_{\max }\right)$, at every pixel, where $T$ was the measured time-varying temperature during the thermal trial. Values of maximum temperature (final $30 \mathrm{~s}$ of laser heating), $T_{\max }$, and ambient temperature (first $5 \mathrm{~s}$ of trial for heating and last $5 \mathrm{~s}$ of trial for cooling), $T_{\mathrm{amb}}$, were calculated at each pixel. Plotting the natural $\log$ of the driving force versus time for a $45 \mathrm{~s}$ cooling period for the AuNP-PDMS films yielded the observed time constant for combined bulk heat transfer (thermal conduction, convection and radiation from film surface) at each pixel of each film.

Expected temperatures were calculated from linear relation of natural log of driving force versus time for comparison with measured temperatures to determine effects of bulk heat transfer. Linear regression from the steady-state portion of the natural log of theta plots was derived for observed theta values, and an expression of the driving force solved for the temperature $(T)$. Predicted values based solely on bulk heat transfer were compared with measured values at a similar time and percent difference taken between the two. Similar calculations were performed for each concentration and numerous regions on each film.

\section{Results and discussion}

\section{Enhanced thermal dissipation immediately after ceasing irradiation}

Nonlinear enhancement of thermal dissipation was qualitatively observed by comparing change in temperature versus time and spatial gradient in dynamic thermal dissipation from the surface of a nanoparticle-containing polymer film (i.e., immediately after laser irradiation had ceased). The rate of thermal dissipation in the thermal micrographs in or near 
the heat spot was observed to be higher than that observed during steady-state heat dissipation a few seconds afterward. This initially amplified dissipation rate decreased superlinearly within seconds after laser irradiation, while surrounding media exhibited lower, predictable steady heat dissipation with little if any observable change. Observations of this phenomenon are shown in Fig. 2, through pixel-by-pixel temperature differences in thermal micrographs $1 \mathrm{~s}$ before and after the laser was shut off in (a) and through the gradient of temperature differences from (a) using Eq. (1) shown in (b). The heat spot (region of interest) was expressed in Fig. 2 as a white square, with AuNP concentration decreasing from A (highest) to F (lowest). Pixels selected in Fig. 2a for subsequent images and analysis were shown as black squares with purple dots for inside and adjacent pixels selected in a controlled manner and as a white square with a black dot for adjacent pixels selected randomly. Figure $2 b$ shows the inside and controlled adjacent selection as white boxes and the randomly selected adjacent pixels as red boxes. Similar thermal micrographs for $16 \mathrm{~nm}$ and $76 \mathrm{~nm}$ AuNP films irradiated at $25 \mathrm{~mW}$ laser power are shown in Figures S2 and S3, respectively. Previous work has spatially analyzed the heating temperature distribution of particles with analysis of experimental data from Au nanorods [27] and simulations of similar rods immersed in water [28].

Enhanced heat transfer coincided with an increase in the temperature change shown in Fig. 2a as well as heat flow away from the heat spot in Fig. 2b, which was readily observed in films A-C. The rate of temperature decrease within and adjacent to the irradiated spot once laser irradiation had ceased was significantly larger than in nonirradiated areas of each sample. This was attributable to thermal decay processes beyond bulk heat transfer via bulk conduction through the PDMS film and convective and radiative heat transfer from the film surface to the surrounding environment. The rate of temperature decrease between 16 and $76 \mathrm{~nm}$ AuNP films at $25 \mathrm{~mW}$ laser power (Figures S2 and S3, respectively) was similarly localized within the heat spot of each respective sample with the $76 \mathrm{~nm}$ AuNP films having lower heat loss than $16 \mathrm{~nm}$ AuNP films. This observable heat loss shown in Fig. 2 was attributed to the rapid heating and cooling of the AuNPs due to the irradiation of the AuNPs with resonant light resulting in heat dissipation propagating through the PDMS matrix as the AuNP plasmons relax. Plasmon relaxation from the AuNPs scatters the absorbed light from the incident wave into embedded PDMS polymer matrix, which allows heat diffusion to propagate at a greater rate through the material than it would have via bulk PDMS heat transfer. Increased scattering was observed in PDMS films containing $76 \mathrm{~nm}$ AuNPs that yielded a lower temperature before and after the laser irradiation had ceased. PDMS films containing $16 \mathrm{~nm}$ AuNPs possess no scattering component according to Mie theory simulations, and thus heat dissipation was greatest in the laser irradiation spot compared to the surrounding polymer matrix and resulted in a loss of enhanced heat dissipation.

Figure $2 \mathrm{a}$ shows that as the concentration of $16 \mathrm{~nm}$ AuNPs increased from film F to film A, there was a corresponding increase in the magnitude and breadth of change in temperature (heat flow around the heat spot), e.g., the number and intensity of yellow to blue pixels in each micrograph increased. Substantial temperature changes within and adjacent to the heat spot observed in Fig. 2 infer the impact of an internal force restricting the overall thermal dissipation of the films. Near-uniform change in the temperature (Fig. 2a) and gradient (Fig. 2b) magnitude for film $F$ indicated an insubstantial response in nanocomposite film after the laser was shut off and within the dissipation of the thermal energy.
Fig. 2 Initial heat dissipation of AuNP-PDMS films after laser shutoff displayed strong diffusion trends confined within and adjacent to the heat spot. Qualitative observation of enhanced heat transfer, as shown through the $\Delta \mathrm{T} 1 \mathrm{~s}$ before and after shutoff shown in (a) and the spatial gradient of the change in temperature from (a) shown in (b). The concentration of $16 \mathrm{~nm}$ AuNPs in each film $(\sim 5 \times 5 \mathrm{~mm})$ decreased from a high value in image $\mathrm{A}$ to a low value in image $\mathrm{F}$ as follows: $\mathrm{A}=0.015 \%, \mathrm{~B}=0.01 \%$, $\mathrm{C}=0.0075 \%, \mathrm{D}=0.005 \%$, $\mathrm{E}=0.002 \%$, and $\mathrm{F}=0.001 \%$ (a)

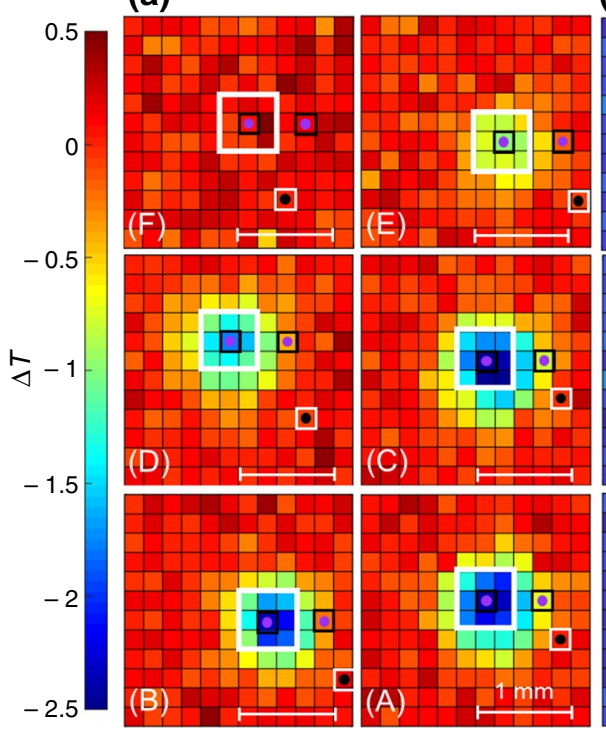

(b)

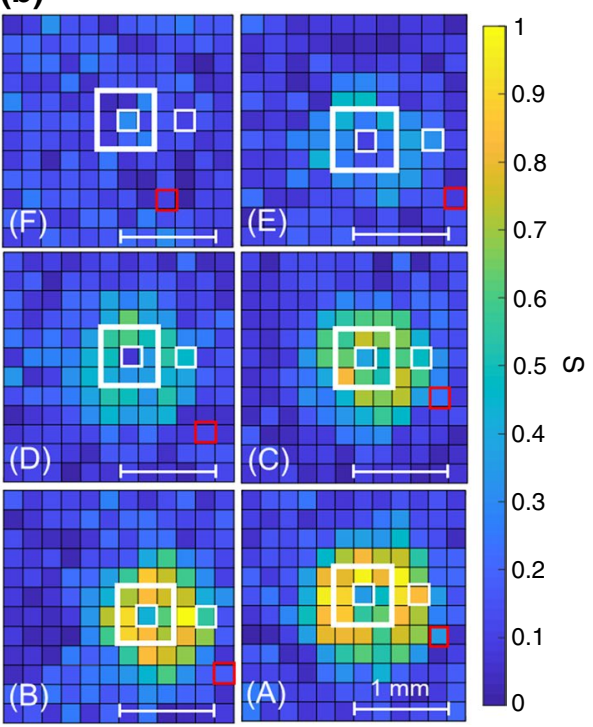


The magnitude of the temperature and gradient increased with concentration of $16 \mathrm{~nm}$ AuNPs but remained closely confined within the heat spot as shown in films A-D.

\section{Dynamic and steady-state thermal dissipation modes}

Enhancement of the thermal dissipation rate was observed as initial, multimodal thermal dynamics in a conventional plot of the natural log of theta versus time from pixels from AuNP-PDMS films in or near the laser spot as shown in Fig. 3a. The temperature driving force (theta) was calculated at every pixel via MATLAB and evaluated inside (Fig. 3b), adjacent to (Fig. 3c), and far outside (Fig. 3d) the heat spot for each film. Figure $3 b-d$ plots the slope of the dynamic thermal mode ( $0-3 \mathrm{~s})$ and the steady-state thermal mode (15-40 s) versus the AuNP concentration for films containing $16 \mathrm{~nm}$ and $76 \mathrm{~nm}$ AuNPs irradiated at $13.5 \mathrm{~mW}$ and $25 \mathrm{~mW}$ laser powers for pixels inside, adjacent to, and outside the heat spot. More obscure multimodal thermal dynamics in $76 \mathrm{~nm}$ compared to $16 \mathrm{~nm}$ AuNP films was a result of reduced thermal effects from insufficient plasmon resonance due to the $532 \mathrm{~nm}$ laser wavelength being $-40 \mathrm{~nm}$ blueshifted from the localized surface plasmon resonance

(a)

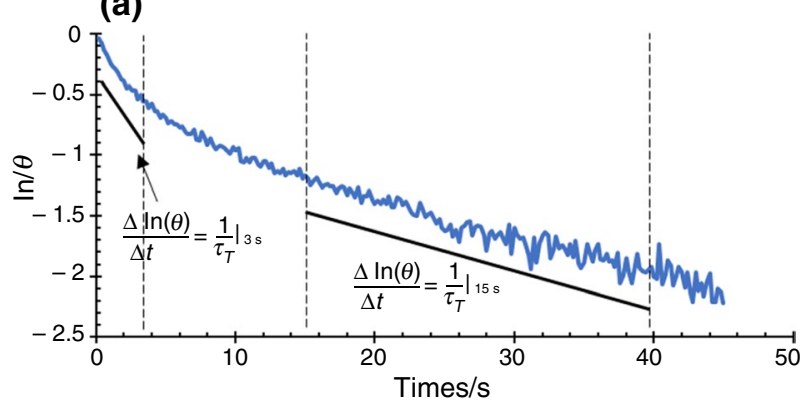

(c)

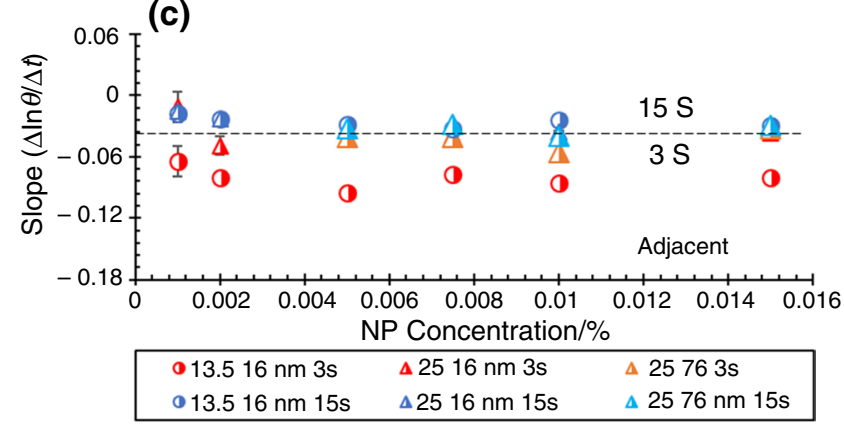

Fig. 3 Irradiated AuNP-PDMS films undergo an initial enhanced, nonmonotonic heat dissipation (dynamic) that decays to a monotonic decrease (steady-state) through the change in the $\ln (\theta)$ with time, according to the pixel location. The rate of change (slope) of $\ln (\theta)$ with time $(\Delta \ln (\theta) / \Delta t)$ was taken at $0-3 \mathrm{~s}$ (dynamic thermal mode) and 15-40 s (steady-state thermal mode) to show this nonmonotonic and monotonic behavior. (a) $\ln (\theta)$ versus time with slopes (black
(LSPR) peak for the $76 \mathrm{~nm}$ AuNP films $(-570 \mathrm{~nm}$, corresponding spectra for $16 \mathrm{~nm}$ and $76 \mathrm{~nm}$ AuNP films shown in Figure S4). Optimal thermal heating of AuNPs occurs at the LSPR wavelength with lower temperature changes observed further from the peak wavelength as a result of lower energy absorption away from the LSPR wavelength. The observed multimodal activity decayed as time progressed before reaching an expected linear relation with time as the system reached the steady-state thermal dynamic mode, similar to previously reported nanocomposite samples [29]. The multimodal activity also decreased as the temperature driving force was evaluated further away from the heat spot

\section{Effects of pixel location}

Figure 3a shows the multimodal activity of the temperature driving force where $0-3 \mathrm{~s}$ shown was the dynamic thermal mode, 15-40 s was the steady-state thermal mode, and 3-15 s was the transition period between the dynamic and steady-state thermal modes. This transition period was not observed to have significant insight into the thermal dynamics of the system and was not used for analysis. The slopes $(\Delta \ln (\theta) / \Delta t)$ of the dynamic and steady-state thermal modes were determined for inside,
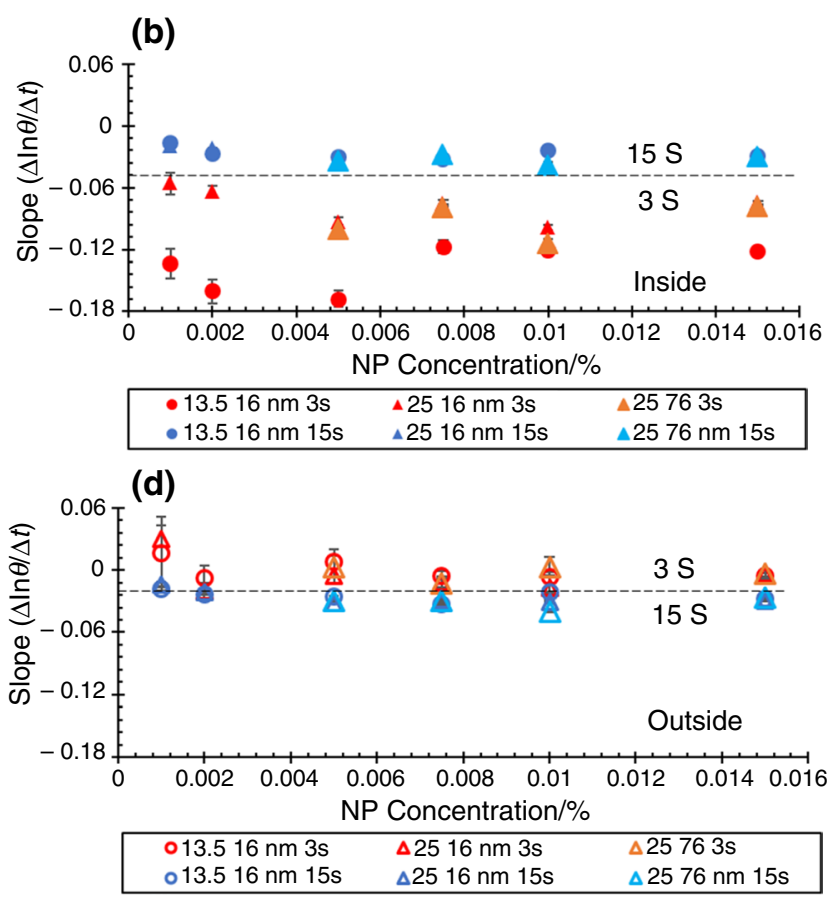

lines) plotted below quantitatively showing enhanced temperature change for (b) $\Delta \ln (\theta) / \Delta t$ inside (In) the heat spot, (c) pixels adjacent (Adj) to the heat spot, and (d) pixel outside (Out) the heat spot. $\Delta \ln (\theta) / \Delta t$ for $0-3 \mathrm{~s}$ (red/orange) and 15-40 s (blue/sky blue) plotted against concentration for $16 \mathrm{~nm}$ AuNPs at $13.5 \mathrm{~mW}$ (circles) and 25 $\mathrm{mW}$ (triangles), and $76 \mathrm{~nm}$ AuNP for dynamic and steady-state thermal modes. Error bars for slope values may be obscured by marker(s) 
adjacent to, and outside of the heat spot for each of the films to determine the impact of the pixel location, AuNP diameter and concentration, and laser power shown in Fig. 3(b-d). The slopes for pixels within the heat spot ranged from -0.05 to -0.17 for the dynamic thermal modes and -0.01 to -0.05 for the steady-state thermal modes with a clear divide between the dynamic and steady-state modes observed in Fig. 3b distinguished via a dashed line at a slope of -0.05 . The slopes for the dynamic thermal mode resulted in approximately tenfold increase compared to the slopes observed for the steadystate thermal mode within the heat spot.

As the pixel selection moved from within the heat spot to adjacent to the heat spot (shown in Fig. 3c), the clear divide observed in Fig. 3b became less clear with several of the dynamic thermal mode slopes crossing the dashed line above -0.05 . When a pixel well outside the heat spot was selected (shown in Fig. 3d), the slopes for the dynamic thermal mode were observed above those of the steady-state thermal mode. Films containing $16 \mathrm{~nm}$ AuNPs irradiated at $13.5 \mathrm{~mW}$ laser power generally resulted in steeper dynamic thermal mode slopes within and adjacent to the heat spot, while slopes outside the heat spot were similar to films containing $16 \mathrm{~nm}$ and $76 \mathrm{~nm}$ AuNPs irradiated at $25 \mathrm{~mW}$. Steady-state thermal modes within, adjacent to, and outside the heat spot resulted in similar slopes between 0 and -0.05 no matter the pixel selection, AuNP diameter, or AuNP concentration, while the dynamic thermal modes varied between 0.05 and -0.17 depending on whether the pixel selected was within, adjacent to, or outside the heat spot. Higher observable dynamic thermal mode slopes suggested additional heat dissipation beyond that of bulk heat transfer occurred within the film.

(a)

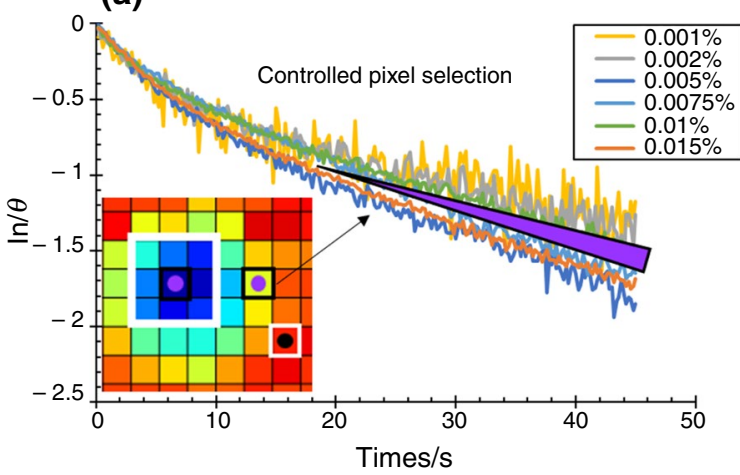

Fig. 4 Selection process (controlled versus random) for selecting pixels adjacent to the heat spot was observed to be correlated with the orientation of the $3 \times 3$ region of interest as shown in the $\ln (\theta)$ versus time data for pixels that were chosen in a controlled manner (a)

\section{Controlled versus random adjacent pixel selection}

The impact of which pixel was selected for evaluation within and outside of the heat spot, respectively, appeared to be minimal, while the selection of the location of pixels adjacent to the heat spot appeared to have a significant impact on the resulting dynamic thermal modes for each of the films. In order to show the effect of the adjacent pixel selection more clearly, two methods for selecting the pixels were used: (1) a controlled process where the pixel selected was in a similar location for each film as shown in Fig. 4 with the black box containing the purple dot to the right of the white box and (2) randomly selecting pixels adjacent to the heat spot where the pixel selected for each film was in a different relative location as shown in Fig. 4 with the white box containing a black dot. The selection of these pixels for analysis resulted in significantly different results between the controlled and random selection processes.

Degree of divergence of $\ln (\theta)$ versus time with concentrations for pixels adjacent to the heat spot supported a correlation to selection of pixels used in data analysis. The method for determining the controlled pixel selection was by taking a $3 \times 3$-pixel array next to the white box outlining the heat spot and selecting the center pixel in that $3 \times 3$ array. This method was used for each film for the controlled pixel selection process resulting in the natural $\log$ of theta versus time plots shown in Fig. 4a. The purple triangle outlined in black shows the divergence in the data with films $\mathrm{A}, \mathrm{C}$, and $\mathrm{D}$ below the triangle and films $\mathrm{B}, \mathrm{E}$, and $\mathrm{F}$ above the triangle. The divergence in Fig. 4a appeared to begin at $-18 \mathrm{~s}$ and continued to diverge at a slow rate until the $45 \mathrm{~s}$ cutoff was reached with the resulting slopes for the dynamic and steady-state thermal modes showing greater correlation between datasets. This pixel selection process was

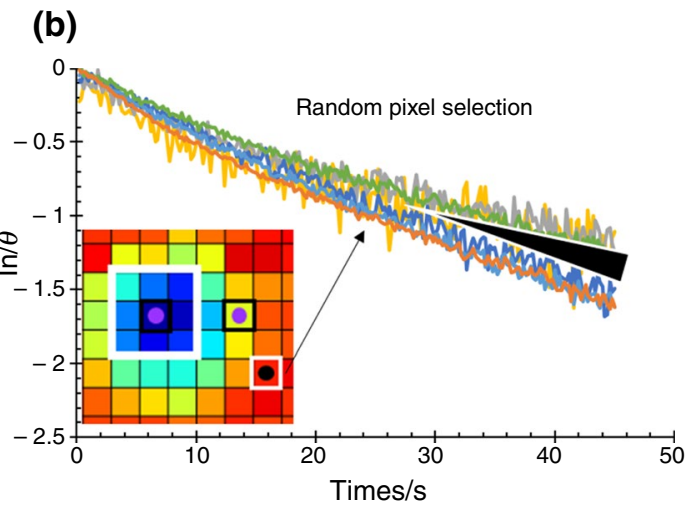

and pixels chosen randomly (b) for $13.5 \mathrm{~mW}$ trials. Triangle in each figure shows divergence or separation in concentration highlighting importance of pixel selection when evaluating thermal effects in nanocomposite samples 
used for all data and analysis throughout this work. Pixels chosen randomly did not show the same strong correlation, but instead showed no definitive features or overall discernable trends, regardless of distance to the heat spot. Evidence of this was shown in the divergence and overlap of natural log of theta versus time curves shown in Fig. 4b. The divergence in Fig. 4b appeared to start at $-30 \mathrm{~s}$ and diverged at a faster rate than the controlled selection in Fig. 4a. The overall shapes of the curves in Fig. 4a are similar as well, while the curves in Fig. 4b vary in shape. The curves generated for the pixels chosen in a controlled manner showed a small, clear distinction between films of varying thicknesses, while pixels chosen randomly showed a large difference and degree of overlap between films with varying thicknesses. These observations showed the importance of selecting pixels in similar locations for performing thermal analysis. Supporting information shows the $\ln (\theta)$ versus time plots for $16 \mathrm{~nm}$ and $76 \mathrm{~nm}$ films plotted at $25 \mathrm{~mW}$ laser power for controlled (Fig. S5) and randomly selected pixels (Fig. S6) for inside, adjacent to, and outside the heat spot.

\section{Effects of laser power}

Increasing continuous laser power from 13.5 to $25 \mathrm{~mW}$ diminished initial heat dissipation and initial temperature elevation in the first $0-10 \mathrm{~s}$ after laser irradiation ceased. Figure 5a shows initial dynamic ( $0-3 \mathrm{~s}$, red) and steadystate (15-45 s, blue) heat dissipation rates, which are proportional to $|\Delta \ln (\theta) / \Delta t|$ termed 'Slope', for $\sim 1.05$-mm-thick films containing $16 \mathrm{~nm}$ AuNPs that were irradiated at 13.5 (circles) and $25 \mathrm{~mW}$ (triangles). Rates for pixels inside (filled symbols), adjacent (half-filled symbols), and outside (open symbols) were distinguished. Heat dissipation of pixels inside and adjacent to the laser spot was damped by irradiation at $25 \mathrm{~mW}$ relative to $13.5 \mathrm{~mW}$. Comparing Fig. $7 \mathrm{~b}$ and $\mathrm{c}$ shows initial temperature elevation of pixels inside and adjacent to the spot was similarly damped at $25 \mathrm{~mW}$ relative to $13.5 \mathrm{~mW}$. This damping appeared due to greater saturation of optoplasmonic heating of AuNP at the higher power. Damping extended to adjacent pixels, ostensibly due to optical diffraction that reoriented resonant irradiation in a radial direction to optoplasmonically heat adjacent AuNP [32]. Heat dissipation of pixels outside the laser spot
Fig. 5 Thin films with fewer NP thermally equilibrate faster. a Dynamic heat dissipation rate (red symbols, $0-3 \mathrm{~s}$ ) which is proportional to $\mid \Delta \ln (\theta) /$ $\Delta t \mid$ decreases as $16-\mathrm{nm}$ AuNP content increases $(0.005$ to 0.01 to $0.015 \%$ ) in $-1.05-\mathrm{mm}$ thick films for pixels inside (filled symbols) and adjacent (half-filled symbols) to the laser spot at $13.5 \mathrm{~mW}$ (circles) and $25 \mathrm{~mW}$ (triangles) irradiation. Heat dissipation rate is nearly constant, approaching zero outside (open symbols) the spot and at steady state (blue symbols, $15-45 \mathrm{~s}$ ) for all 16-nm AuNP contents. b Thicker films $(\sim 1.40 \mathrm{~mm})$ with low $(0.0075 \%)$ AuNP have dynamic heat dissipation rates inside/ adjacent to (filled/half-filled) the heat spot that are nearly equal for $16 \mathrm{~nm}$ (red) and $76 \mathrm{~nm}$ (orange) AuNP but lower than $1.05 \mathrm{~mm}$ films. Steady-state dissipation rates (blue symbols) are comparable to those for $1.05 \mathrm{~mm}$ films for both $16 \mathrm{~nm}$ and $76 \mathrm{~nm}$ AuNP. Error bars for slope values may be obscured by marker(s) (a)
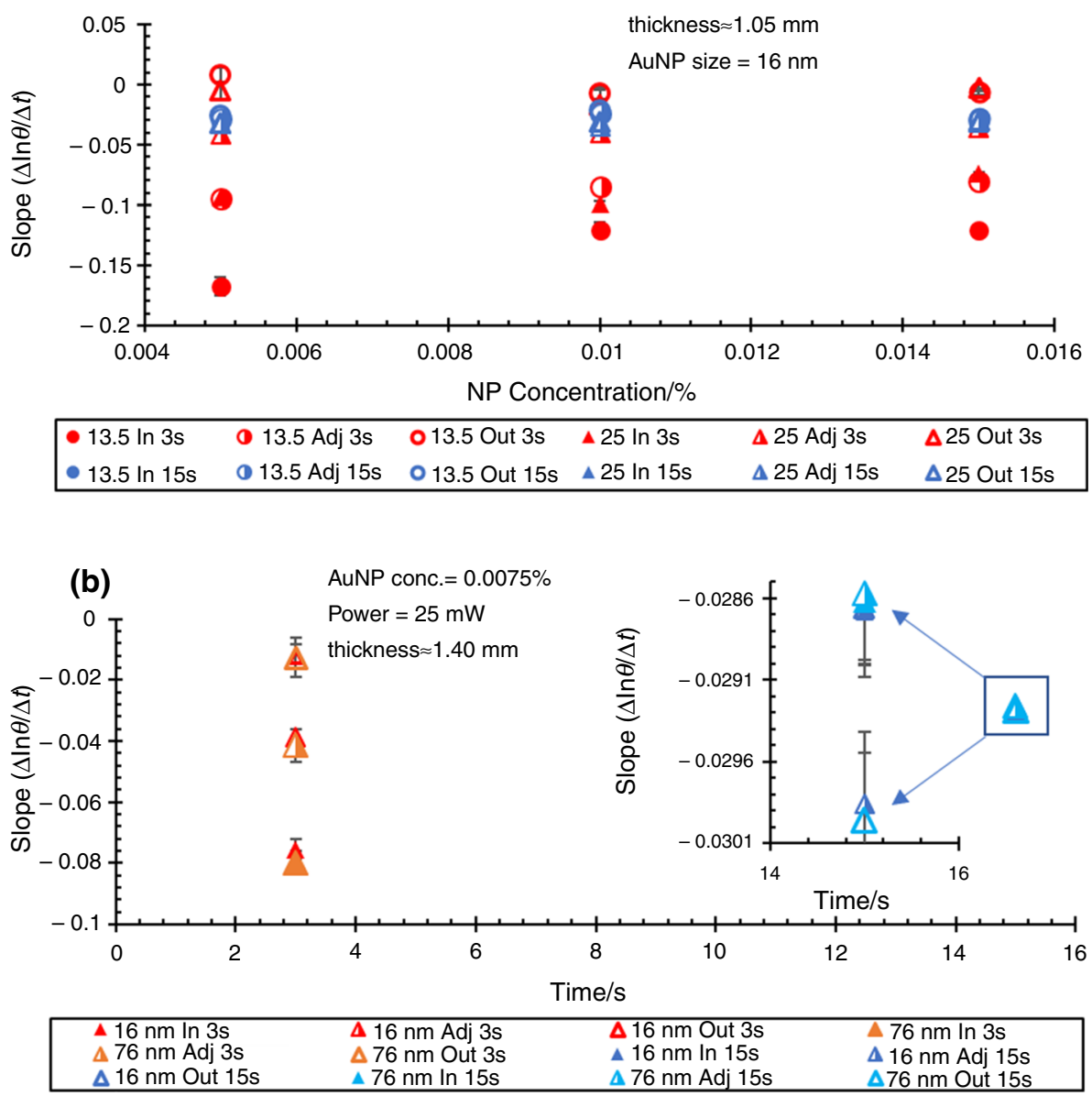
was lower still, remained relatively constant, and appeared unaffected by laser power. Such a result is consistent with putative saturation of optoplasmonically heated AuNP inside and adjacent to the laser spot at higher resonant irradiation power. Effects of laser power herein are comparable to those of Garwe et al., who reported control of the cooling response from pulsed irradiation and laser power for AuNPs [30]. Greater laser powers result in longer time elapse between particle heating and cooling.

Steady-state heat dissipation rates (blue symbols) throughout all films were nearly identical and lower than initial values for inside or adjacent pixels. Dynamic dissipation had ended in the first $10 \mathrm{~s}$; by $15-45 \mathrm{~s}, \log$-linear heat dissipation occurred via linear conduction and convection and pseudolinear radiation [16]. Increasing laser power had no visible effect on steady heat dissipation. Putative effects of saturation appeared only in the initial dynamic period in pixels containing resonantly irradiated AuNP.

\section{Effects of AuNP concentration}

Thermal dissipation within AuNP-PDMS films was enhanced at lower concentrations of AuNPs from a greater thermal gradient between the hot AuNPs and surrounding PDMS. Films with comparable thickness $(\sim 1.05 \mathrm{~mm})$ containing $16 \mathrm{~nm}$ AuNPs with concentrations of $0.005 \%$, $0.01 \%$, and $0.015 \%$, shown in Fig. 5a displayed a range of dynamic mode slopes from -0.17 to 0.0077 , which were observed to increase in magnitude as pixels closer to the heat spot were measured. Steady-state thermal dissipation slopes were comparable for all concentrations at $\sim-0.025$. With the thermal measurements performed on a pixel-by-pixel basis, each analyzed pixel consisted of a combination of AuNPs and PDMS. This led films at lower AuNP concentrations to have a higher PDMS content (strong insulator) and a lower AuNP content (strong conductor) in each pixel. This resulted in a larger temperature driving force for thermal dissipation due to the combination of AuNPs and PDMS within each pixel area. AuNPs irradiated at resonant frequencies reached temperatures much higher than the surrounding PDMS, resulting in increases in the overall average measured composite matrix (AuNPs + PDMS) surface temperatures achieved during steady-state irradiation. Films containing a higher number of AuNPs per pixel (i.e., larger AuNP concentration) heated the immediately adjacent PDMS to a greater temperature than films with a lower concentration. Greater differences between the PDMS and adjacent AuNPs result in a larger thermal gradient between the PDMS matrix from the hot AuNPs, which resulted in a higher, but rapidly damped, rate of thermal dissipation in that area of the film. When transitioning from the dynamic thermal mode to the steady-state thermal mode, the effect diminished, resulting in similar slope values across all concentrations, laser powers, and pixel locations as shown in Fig. 5.

A smaller transitional change from dynamic to steadystate thermal mode appears due to increased electron-phonon relaxation at greater AuNP concentrations. AuNP films containing $16 \mathrm{~nm}$ AuNPs irradiated at $13.5 \mathrm{~mW}$ showed a decreasing slope change inside the heat spot from approximately $-0.17,-0.121$, and -0.122 in dynamic mode to -0.0320 in steady-state mode as concentration increased from 0.005 to $0.015 \%$. Results for pixels adjacent and outside to the heat spot showed similar slope changes for both films irradiated at both powers. Greater changes in the driving force $(\theta)$ with time can be a result of greater electron-phonon relaxation of the AuNPs within the material. Shahariari et al. showed that thermal diffusivity of a material increased with concentration from greater electron-phonon relaxation and subsequent phonon scattering at the nanoparticle media interface. Larger thermal diffusivities allow for increased thermal propagation, thus implying faster changes from the dynamic driving force to the steady-state driving force [31]. Additionally, Howard et al. demonstrated a plateau of thermal energy transfer from AuNPs, which allows for lower concentration AuNP-PDMS films to have a greater change in slope at a smaller concentration than for larger AuNP concentrations, matching measured results [20].

\section{Effects of film thickness}

The thin, optically transparent, thermally insulating PDMS films slowed conductive heat dissipation in proportion to thickness as calculated by the Biot ratio [26]. Figure 6 shows average heat dissipation rate, which is proportional to

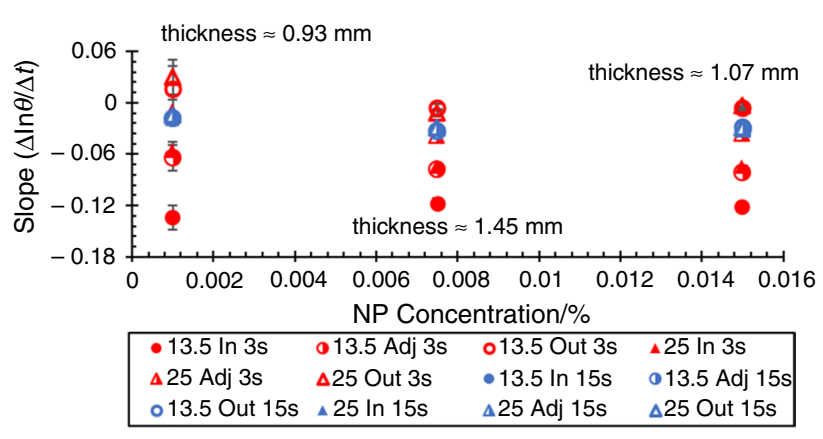

Fig. 6 Thicker films with more AuNP thermally equilibrate slower inside the laser spot. a Dynamic heat dissipation rate (red symbols, $0-3 \mathrm{~s})$ which is proportional to $|\Delta \ln (\theta) / \Delta t|$ decreased inside (filled symbols) the laser spot at $13.5 \mathrm{~mW}$ (circles) for $16 \mathrm{~nm}$ AuNP films in the order $0.93 \mathrm{~mm}>1.07 \mathrm{~mm}>1.45 \mathrm{~mm}$ across AuNP contents from 0.001 to 0.0075 to $0.015 \%$. Pixels adjacent to (half-filled symbols) or outside (open symbols) the spot equilibrate faster as thickness and AuNP content increase. Decreasing thermal gradients drive both observations. Error bars for slope values may be obscured by marker(s) 
$|\Delta \ln (\theta) / \Delta t|$, decreased in $13.5 \mathrm{~mW}$ laser-irradiated pixels (in red filled circles) of 16-nm AuNP-PDMS films as thickness increased from 0.93 to 1.07 to $1.45 \mathrm{~mm}$ within $3 \mathrm{~s}$ (dynamic mode) after laser irradiation ceased. This was attributable to rapid radiative cooling of a small number of optothermally superheated AuNP insulated in a cooler PDMS matrix, whose effect decreased for thicker films with more AuNP. Three factors-higher power ( $25 \mathrm{~mW}$, triangles), distance from the laser spot, and AuNP concentration-mitigated this trend. Dynamic heat dissipation rates in the spot at $25 \mathrm{~mW}$ (red filled triangles) were lower than in-spot pixels at $13.5 \mathrm{~mW}$, with rates similar to pixels adjacent (red half-filled circles) to the spot at $13.5 \mathrm{~mW}$. These rates remained relatively constant with increasing AuNP content from 0.001 to $0.015 \%$ and increasing thickness. Lower rates at $25 \mathrm{~mW}$ than $13.5 \mathrm{~mW}$ appeared due to more saturation of optothermal NP heating at $25 \mathrm{~mW}$. Values of rates in adjacent pixels at $13.5 \mathrm{~mW}$ intermediate between pixels inside and outside the laser spot were attributable to optical diffraction that heated $16 \mathrm{~nm}$ AuNP adjacent to the spot to a lesser, but finite, degree compared to AuNP within the spot [32]. Dynamic rates outside the spot at $0.001 \%$ (open red symbols) were initially positive as energy was conducted into them from in-spot pixels immediately after radiation ceased. All other pixels had marginally higher rates proportional to thickness in the order $1.45 \mathrm{~mm}>1.07 \mathrm{~mm}>0.93 \mathrm{~mm}$ as more insulation increased initial bulk temperature (see Fig. 7) and maintained a higher dissipation rate over a longer period. This included heat dissipation rates measured at steady state (15-45 s, blue symbols) for pixels inside (filled symbols) adjacent (half-filled symbols) and outside (open symbols) the laser spot at both $13.5 \mathrm{~mW}$ (circles) and $25 \mathrm{~mW}$ (triangles). These observed effects of film thickness reducing thermal dynamics matched previously reported results [26].

\section{Effects of AuNP dispersed in polymer on temperature driving force for thermal dissipation}

Measured initial temperatures $(T)$ of resonantly irradiated AuNP-containing polymer were elevated relative to temperature values anticipated by log-linear steady-state heat diffusion. Figure 7a compares measured (red dot/blue solid line) temperatures relative to values predicted (green dot/ black dashed line) from a least-squares fit (inset) to the (a)
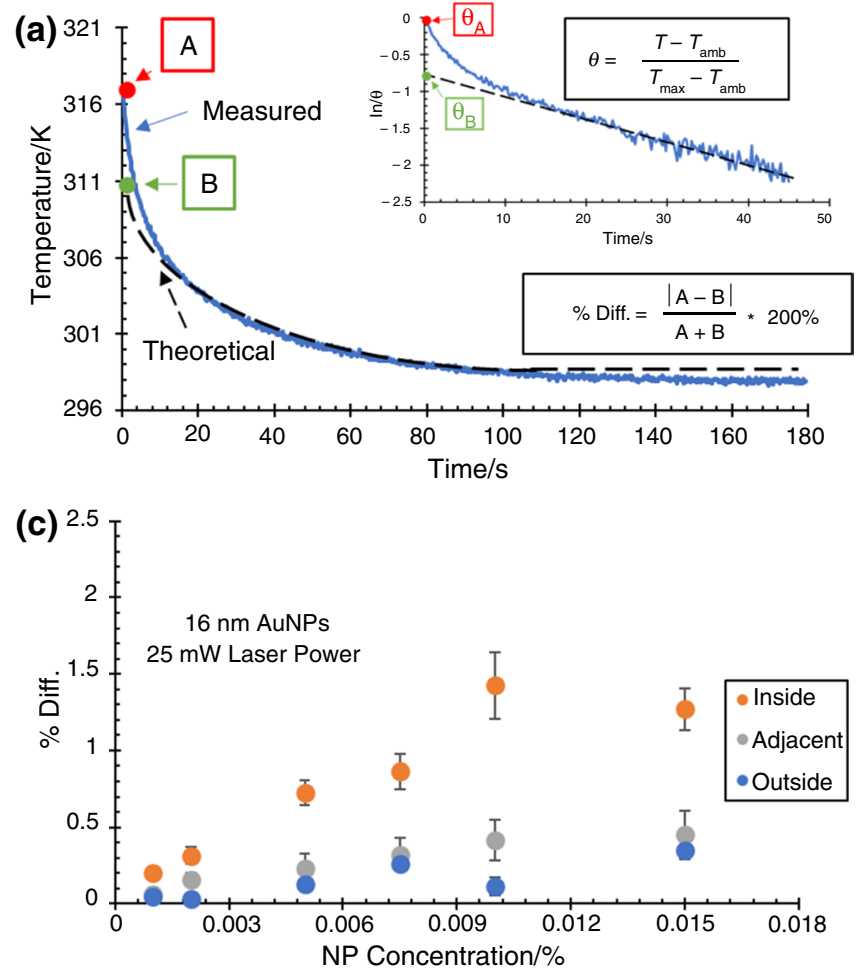

Fig. 7 More concentrated AuNPs enhance multiexponential heat dissipation in pixels irradiated inside (orange) the laser spot, whose effect is reduced in pixels adjacent to (gray) or outside (blue) the spot. Measured temperature when laser is extinguished exceeds that predicted by a monoexponential model of linear heat transfer via radiation, convection and conduction. a Temperature versus time for measured (red dot/blue solid line) and theoretical (green dot/black dashed
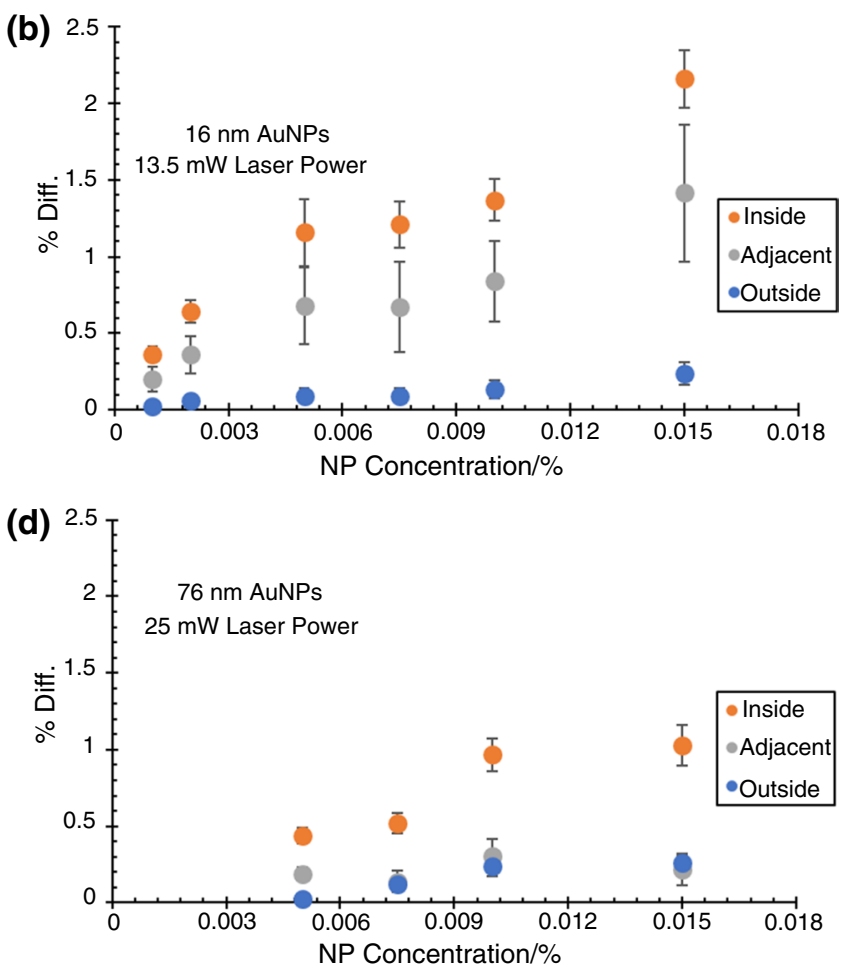

line). Inset shows semilog plot of scaled temperature $(\theta)$ to linearly predicted monoexponential initial temperature. The higher the initial temperature, the faster the heat dissipation, and multiexponential temperature decay is largest for b $16 \mathrm{~nm}$ AuNP films at $13.5 \mathrm{~mW}$, and lesser for c $16 \mathrm{~nm}$ AuNP at $25 \mathrm{~mW}$ and d $76 \mathrm{~nm} \mathrm{AuNP}$ at $25 \mathrm{~mW}$. Error bars for slope values may be obscured by marker(s) 
semilogarithmic plot of dimensionless temperature driving force $(\theta)$ versus time. The fit was based on a continuum model of thermal dissipation driven by a logarithmic decrease in temperature driving force $(\theta)$ due to linear conduction, convection, and linearized radiative heat transfer [16]. This model, illustrated in Fig. 7a inset, describes steady heat dissipation from the nanocomposite AuNP-PDMS films suitably from ca. $10 \mathrm{~s}$ onward. At time values preceding $10 \mathrm{~s}$, $\ln (\theta)$ decreased superlinearly as $T$ decreased multiexponentially from an initial value (red dot) measured immediately after laser irradiation ceased. Measured initial values of $\theta_{\mathrm{A}}$ and $T_{\mathrm{A}}$ (red dots) were compared relative to those anticipated by the log-linear model $\left(\theta_{\mathrm{B}}\right.$ and $T_{\mathrm{B}}$-green dots) from 15 to ca. $40 \mathrm{~s}$. Temperature elevation in the first few seconds appeared due to the nanocomposite nature of the films: A small fraction of AuNPs ( $\leq 0.015$ mass\% for 16-nm NP) were heated optoplasmonically to temperatures much hotter than intervening PDMS. Thermal images recorded an average temperature at each pixel consisting a small number of directly heated 'hot spots' at each AuNP and a much larger ( $\geq 6666$-fold for $16 \mathrm{~nm} \mathrm{NP}$ ) mass of insulating PDMS heated primarily by conduction

Comparison of the initial temperature was quantified by calculating the percent difference (\% Diff) between measured and predicted temperature. Specifically, the percent difference between measured and anticipated temperature was determined as $\%$ Diff $=\frac{\left|T_{\mathrm{A}}-T_{\mathrm{B}}\right|}{T_{\mathrm{A}}+T_{\mathrm{B}}} * 200 \%$, where $T_{\mathrm{A}}$ was the measured temperature from thermal excitation and $T_{\mathrm{B}}$ was the temperature obtained by linearly extrapolating $\ln (\theta)$ versus time in steady-state thermal mode back to $t=0 \mathrm{~s}$, as shown in Fig. 7a inset.

The initial increase in temperature driving force trended higher for directly irradiated $16 \mathrm{~nm}$ AuNP-PDMS composite films with higher mass\% Au. Figure $7 \mathrm{~b}$ shows \% Diff for $16 \mathrm{~nm}$ AuNP-PDMS films irradiated at $13.5 \mathrm{~mW}$. Measured initial temperature exceeded the back-extrapolated value by $0.36 \%$ to $2.16 \%$ in pixels inside the laser spot, $0.13 \%$ to $1.20 \%$ in pixels adjacent to the laser spot, and $0.033 \%$ to $0.40 \%$ in pixels outside the laser spot. Similar trends albeit smaller in magnitude were observed for $16 \mathrm{~nm}$ AuNPs-PDMS and $76 \mathrm{~nm}$ AuNP-PDMS irradiated at $25 \mathrm{~mW}$, as shown in Fig. 7c and d, respectively. Specifically, difference values for pixels within the heat spot increased from 0.21 to $1.69 \%$ for $16 \mathrm{~nm}$ AuNP at $25 \mathrm{~mW}$, and $0.49 \%$ to $1.13 \%$ for $76 \mathrm{~nm}$ AuNPs. Overall, it appeared that increased mass\% of AuNP raised the contribution of 'hot AuNP spots' to average temperature measured for each pixel in the thermal image, thus increasing the initial temperature elevation.

Irradiating $16 \mathrm{~nm}$ AuNP-PDMS at $13.5 \mathrm{~mW}$, as in Fig. 7b, yielded initial temperature increases generally higher than irradiation at $25 \mathrm{~mW}$, as in Fig. 7c. This increase appeared due to a greater degree of optical saturation of the irradiated AuNP at the higher power.

Measured initial temperature elevation decreased for pixels further from the laser-irradiated spot. Comparing Fig. 7b-d shows initial temperature differences were less than $0.4 \%$ in pixels outside the laser spot for all AuNP concentrations irradiated at all powers. Initial temperature elevation was larger for pixels adjacent to the heat spot. Percent differences rose from ca. 0.1 to $1.4 \%$ across all films, largely in proportion to increases in AuNP mass\%. Marked initial temperature elevation in pixels of $16 \mathrm{~nm}$ AuNP films adjacent to the laser spot appeared due to irradiation of AuNP in these pixels via optical diffraction, by which irradiation normal to the film is radially redirected. [32] Increased AuNP content affected initial temperature elevation the most in adjacent pixels. Apparent saturation of $16 \mathrm{~nm}$ AuNP at $25 \mathrm{~mW}$ lessened the affect, as shown in Fig. 7c. Increasing AuNP diameter to $76 \mathrm{~nm}$ further reduced initial temperature elevation in adjacent pixels, as shown in Fig. 7d, even though larger AuNP can scatter radiation radially. However, larger particles at similar mass\% concentration corresponded to less scattering as well as a smaller number of 'hot AuNP spots', thus reducing initial temperature elevation inside and adjacent to the laser spot.

Deviations from largely monotonic increases in measured initial temperature elevation proportional to AuNP concentration appeared due to host matrix thermal properties for 16-nm AuNP-PDMS films and nonresonant plasmon irradiation for $76 \mathrm{~nm}$. Figure $7 \mathrm{~b}$ and d shows monotonic increases for nearly all percent difference values at all pixel locations, while Fig. 7c shows monotonic increases in percent differences for adjacent pixels and inside pixels at concentration $\leq 0.01 \%$. Nonmonotonic increases in percent difference are observed at $0.01 \%$ for pixels outside the laser spot in Fig. 7c. These pixels were heated by bulk thermal conduction, lacking optoplasmonic heating by direct or scattered laser light. Low conduction in insulative PDMS $\left(-0.15-0.18 \mathrm{~W} \mathrm{~m}^{-1} \mathrm{~K}^{-1}\right)[34,35]$ limited initial temperature values as well as heat dissipation, resulting in small percent differences across all AuNP concentrations. Sample-to-sample variations in PDMS thickness and uniformity may also contribute to nonmonotonicity. For $76 \mathrm{~nm}$ AuNP-PDMS films, in contrast, \% Diff was similar for pixels adjacent to and outside the laser spot. This appeared due to relatively fewer large, scattering AuNPs in tandem with rapid heating and cooling of AuNP-containing media. Despite occasional deviations, initial temperature elevation largely increased monotonically in proportion to AuNP concentration, with faint effects from PDMS thickness and uniformity. It appeared that any sample-to-sample variations in thickness and uniformity did not perceptibly alter dynamic thermal changes in the initial seconds after laser irradiation. 
Effects of AuNP dispersed in polymer on temperature driving force for thermal dissipation can be summarized as follows. Initial temperature elevation and superlogarithmic temperature decreases in resonantly irradiated pixels were attributable to AuNP optoplasmonically heated to temperature value much higher than the bulk of intervening, insulating PDMS. Initial dissipation of AuNP-associated heat occurred primarily by radiation, which varies to the fourth power with temperature. Rapid radiative cooling of AuNPs decreased $\ln (\theta)$ superlinearly until thermal diffusion occurred at a rate governed by the PMDS bulk value, at which point heat dissipation followed the linearized relation. Pixels well outside the laser spot were not directly irradiated by incident resonant light. Such pixels largely lacked the bimodal temperature disparity between AuNP and bulk PDMS and dissipated heat primarily by pseudolinear radiation and linear convection and conduction. Pixels adjacent to the laser spot exhibited multiexponential behavior due to irradiation via optical diffraction induced by AuNP separations that approach the Wigner-Seitz radius. Such diffraction reorients incident light parallel to the film [32]. Diffraction due to AuNPs increased with the mass $\%$ of optically absorbing $16 \mathrm{~nm}$ AuNPs at lower incident power (13.5 mW). Higher incident power appeared to saturate the AuNPs, diminishing the diffraction-induced light reorientation and corresponding multiexponential temperature decay of pixels adjacent to the laser spot [33]. Relatively fewer $76 \mathrm{~nm}$ AuNP separated by distances much greater than the Wigner-Seitz radius exhibited behavior similar to $16 \mathrm{~nm}$ AuNPs irradiated at $25 \mathrm{~mW}$ as a result of scattering.

\section{Conclusions}

This work showed that initial temperature and corresponding heat dissipation in or near a resonantly irradiated spot in nanocomposite AuNP-polymer films were enhanced as a function of film geometry, nanoparticle diameter and concentration, laser irradiation power, and location within and adjacent to the irradiated spot. Within the irradiated laser zone, large temperature gradients localized around a small mass fraction of superheated AuNP drove faster heat dissipation relative to cooler intervening polymer matrix. This resulted in higher average initial temperatures proportional to AuNP number content and faster overall heat dissipation in the first few seconds. Within ca. $10 \mathrm{~s}$, heat dissipation and temperature decayed multiexponentially to constant, conventional dissipation rate and logarithmically decreasing temperature as the 'hot AuNP spots' equilibrated with surrounding polymer. Similar behavior was resulted adjacent to the laser spot due to diffraction induced by smaller AuNP, particularly at high mass\%. Further outside the irradiated zone, there were no hot spots and heat dissipation was predictably constant with logarithmic temperature decay. In thin films with few AuNP, these effects were amplified as temperature gradients increased. Thicker insulating films with more AuNP and/or higher laser power decreased the gradient and diminished the effect. Higher initial (dynamic) multiexponential dissipation appeared most influenced by film thickness and pixel location.

Acknowledgements This work was supported in part by NSF CBET1134222, NSF ECCS-1006927, the University of Arkansas Foundation, and the Walton Family Charitable Foundation. This work was also supported in part by the Center for Advanced Surface Engineering (CASE), under the National Science Foundation (NSF) Grant No. OIA-1457888 and the Arkansas EPSCoR Program. D. K. Roper also acknowledges support from Utah State University. Any opinions, findings, and conclusions or recommendations expressed in this material are those of the authors and do not necessarily reflect the views of the National Science Foundation. The authors would like to thank Caitlyn Chambers for performing initial optothermal measurements for $25 \mathrm{~mW}$ laser powers and Dr. Jeremy Dunklin for fabricating films used in this manuscript.

Author contributions T. V. Howard conducted optothermal measurements, analyzed $3.5 \mathrm{~mW}$ laser power data, and drafted figures and sections of text. K. Berry Jr. analyzed $25 \mathrm{~mW}$ laser power data, refined figures, and drafted and revised sections of text. D. K. Roper derived the approach, directed the work, analyzed the data, and finalized the manuscript text and figures.

\section{Compliance with ethical standard}

Conflict of interest There is no conflict of interest to declare.

Open Access This article is licensed under a Creative Commons Attribution 4.0 International License, which permits use, sharing, adaptation, distribution and reproduction in any medium or format, as long as you give appropriate credit to the original author(s) and the source, provide a link to the Creative Commons licence, and indicate if changes were made. The images or other third party material in this article are included in the article's Creative Commons licence, unless indicated otherwise in a credit line to the material. If material is not included in the article's Creative Commons licence and your intended use is not permitted by statutory regulation or exceeds the permitted use, you will need to obtain permission directly from the copyright holder. To view a copy of this licence, visit http://creativecommons.org/licenses/by/4.0/.

\section{References}

1. Berry KR, Russell AG, Blake PA, Roper DK. Gold nanoparticles reduced in situ and dispersed in polymer thin films: optical and thermal properties. Nanotechnology. 2012;23:11.

2. Bozlar M, He D, Bai J, Chalopin Y, Mingo N, Volz S. Carbon nanotube microarchitectures for enhanced thermal conduction at ultralow mass fraction in polymer composites. Adv Mater. 2010;22:1654-8.

3. Dunklin JR, Forcherio GT, Berry KR, Roper DK. Asymmetric reduction of gold nanoparticles into thermoplasmonic polydimethylsiloxane thin films. ACS Appl Mater Interfaces. 2013;5:8457-66. 
4. Li T-L, Hsu SL-C. Enhanced thermal conductivity of polyimide films via a hybrid of micro-and nano-sized boron nitride. J Phys Chem B. 2010;114:6825-9.

5. Urban AS, Carretero-Palacios S, Lutich AA, Lohmüller T, Feldmann J, Jäckel F. Optical trapping and manipulation of plasmonic nanoparticles fundamentals, applications and perspectives. Nanoscale R Soc Chem. 2014;6:4458.

6. Ishii S, Sugavaneshwar RP, Nagao T. Titanium nitride nanoparticles as plasmonic solar heat transducers. J Phys Chem C. 2016;120:2343-8.

7. Wang S, Riedinger A, Li H, Fu C, Liu H, Li L, et al. Plasmonic copper sulfide nanocrystals exhibiting near-infrared photothermal and photodynamic therapeutic effects. ACS Nano. 2015;9:1788-800.

8. Thepudom T, Lertvachirapaiboon C, Shinbo K, Kato K, Kaneko F, Kerdcharoen T, et al. Surface plasmon resonance-enhanced photoelectrochemical sensor for detection of an organophosphate pesticide chlorpyrifos. MRS Commun. 2018;8:107-12.

9. Wang X, He Y, Liu X, Shi L, Zhu J. Investigation of photothermal heating enabled by plasmonic nanofluids for direct solar steam generation. Sol Energy. 2017;157:35-46. https://doi. org/10.1016/j.solener.2017.08.015.

10. Chen M, Wu Y, Song W, Mo Y, Lin X, He Q, et al. Plasmonic nanoparticle-embedded poly(: p-phenylene benzobisoxazole) nanofibrous composite films for solar steam generation. Nanoscale R Soc Chem. 2018;10:6186-93.

11. Dixon SC, Peveler WJ, Noor N, Bear JC, Parkin IP. Superhydrophobic Au/polymer nanocomposite films via AACVD/swell encapsulation tandem synthesis procedure. RSC Adv R Soc Chem. 2016;6:31146-52.

12. Hoepfner MP, Roper DK. Describing temperature increases in plasmon-resonant nanoparticle systems. J Therm Anal Calorim. 2009;98:197-202.

13. Lalisse A, Tessier G, Plain J, Baffou G. Plasmonic efficiencies of nanoparticles made of metal nitrides ( $\mathrm{TiN}, \mathrm{ZrN}$ ) compared with gold. Sci Rep Nat Publ Group. 2016;6:1-11. https://doi. org/10.1038/srep38647.

14. Ahn W, Zhao X, Hong Y, Reinhard BM. Optoplasmonic networks with morphology-dependent near- and far-field responses. MRS Commun. 2015;5:579-86.

15. Davis-Wheeler Chin C, Akbarian-Tefaghi S, Reconco-Ramirez J, Wiley JB. Rapid microwave synthesis and optical activity of highly crystalline platinum nanocubes. MRS Commun. 2018;8:71-8.

16. Roper DK, Ahn W, Hoepfner M. Microscale heat transfer transduced by surface plasmon resonant gold nanoparticles. J Phys Chem C. 2007;111:3636-41.

17. Chen J, Walther JH, Koumoutsakos P. Ultrafast cooling by covalently bonded graphene-carbon nanotube hybrid immersed in water. Nanotechnology. 2016;27:465705.

18. Ahn W, Roper DK. Transformed gold Island film improves lightto-heat transduction of nanoparticles on silica capillaries. J Phys Chem C. 2008;112:12214-8.

19. Alawi OA, Mallah AR, Kazi SN, Sidik NAC, Najafi G. Thermophysical properties and stability of carbon nanostructures and metallic oxides nanofluids: experimental approach. J Therm Anal Calorim. 2019;135:1545-62. https://doi.org/10.1007/s1097 3-018-7713-x.

20. Howard TV, Dunklin JR, Forcherio GT, Roper DK. Thermoplasmonic dissipation in gold nanoparticle-polyvinylpyrrolidone thin films. RSC Adv R Soc Chem. 2017;7:56463-70.
21. Moraveji MK, Barzegarian R, Bahiraei M, Barzegarian M, Aloueyan A, Wongwises S. Numerical evaluation on thermal hydraulic characteristics of dilute heat-dissipating nanofluids flow in microchannels comparison of different models. J Therm Anal Calorim. 2019;135:671-83. https://doi.org/10.1007/s1097 3-018-7181-3.

22. Berry KR, Dunklin JR, Blake PA, Roper DK. Thermal dynamics of plasmonic nanoparticle composites. J Phys Chem C. 2015;119:10550.

23. Samsonov VM, Bembel AG, Kartoshkin AY, Vasilyev SA, Talyzin IV. Molecular dynamics and thermodynamic simulations of segregation phenomena in binary metal nanoparticles. J Therm Anal Calorim. 2018;133:1207-17.

24. Moradi A, Toghraie D, Isfahani AHM, Hosseinian A. An experimental study on MWCNT-water nanofluids flow and heat transfer in double-pipe heat exchanger using porous media. J Therm Anal Calorim. 2019. https://doi.org/10.1007/s10973-019-08076-0.

25. Frivaldsky M, Pavelek M. Development of temperature stabilization system for biological sample's microscope. J Therm Anal Calorim. 2019. https://doi.org/10.1007/s10973-019-08132-9.

26. Keith Roper D, Berry KR, Dunklin JR, Chambers C, Bejugam V, Forcherio GT, et al. Effects of geometry and composition of soft polymer films embedded with nanoparticles on rates for optothermal heat dissipation. Nanoscale. 2018;10:11531-43.

27. Maity S, Wu W-C, Tracy JB, Gundogdu K, Bochinski JR, Clarke LI. Spatial temperature mapping with polymer nanocomposites undergoing ultrafast photothermal heating via gold nanorods. Nanoscale. 2014;6:15236-47.

28. Ekici O, Harrison RK, Durr NJ, Eversole DS, Lee M, Ben-Yakar A. Thermal analysis of gold nanorods heated with femtosecond laser pulses. J Phys D Appl Phys. 2008;41:185501.

29. Russell AG, McKnight MD, Sharp AC, Hestekin JA, Roper DK. Gold nanoparticles allow optoplasmonic evaporation from open silica cells with a logarithmic approach to steady-state thermal profiles. J Phys Chem C. 2010;114:10132-9.

30. Garwe F, Bauerschäfer U, Csaki A, Steinbrück A, Ritter K, Bochmann A, et al. Optically controlled thermal management on the nanometer length scale. Nanotechnology. 2008;19:055207.

31. Shahriari E, Moradi M, Raeisi M. An experimental study of thermal diffusivity of Au nanoparticles :effects of concentration particle size. J Theor Appl Phys. 2016;10:259-63.

32. Dunklin JR, Forcherio GT, Keith Roper D. Gold nanoparticlepolydimethylsiloxane films reflect light internally by optical diffraction and Mie scattering. Mater Res Express. 2015;2:085005.

33. Chu SW, Su TY, Oketani R, Huang YT, Wu HY, Yonemaru Y, et al. Measurement of a saturated emission of optical radiation from gold nanoparticles: application to an ultrahigh resolution microscope. Phys Rev Lett. 2014;112:1-5.

34. Erickson D, Sinton D, Li D. Joule heating and heat transfer in poly(dimethylsiloxane) microfluidic systems. Lab Chip. 2003;3:141-9.

35. Zhang YF, Ren YJ, Guo HC, Bai SL. Enhanced thermal properties of PDMS composites containing vertically aligned graphene tubes. Appl Therm Eng. 2019;150:840-8.

Publisher's Note Springer Nature remains neutral with regard to jurisdictional claims in published maps and institutional affiliations. 